\title{
Pacman heart coexisting with coarctation of aorta and bicuspid aortic valve
}

\author{
Ferdi KAHRAMAN ${ }^{1}$, Metin Çă̆daş², Aziz Inan Celik ${ }^{3}$, TAHİR BEZGINN${ }^{4}$, and Tezcan \\ Peker $^{5}$ \\ ${ }^{1}$ Gebze Fatih State Hospital Department of Cardiology Gebze Kocaeli TR 41400 \\ ${ }^{2}$ Kafkas Universitesi \\ ${ }^{3}$ Gebze Fatih State Hospital \\ ${ }^{4}$ Kosuyolu Kartal Heart Education and Research Hospital \\ ${ }^{5}$ Private Doruk Yildirim Hospital, Bursa, Turkey
}

October 20, 2020

\begin{abstract}
Coarctation of the aorta (CoA) is a well-known congenital heart disease (CHD), which is often associated with several other cardiac and vascular anomalies, such as bicuspid aortic valve (BAV) and ventricular septal defect. BAV is the most common CHD whereas Pacman heart (incomplete muscular ventricular septal defect) is rare congenital deformity. We found a coexistence of Pacman heart, CoA and BAV in a 21- year- old male patient presenting with headache and high blood preesure. This extremely rare combination of these three congenital cardiac abnormalities occurring in the same patient has not been reported yet.
\end{abstract}

\section{Pacman heart coexisting with coarctation of aorta and bicuspid aortic valve}

A 21-year-old male patient presented with headache and hypertension $(160 / 95 \mathrm{mmHg})$ and his clinical history was unremarkable. On physical examination, diastolic murmur was detected in the parasternal 3rd and 4th intercostal region. In transthoracic echocardiography, left ventricle diameters were within normal range, ejection fraction was preserved and moderate eccentric aortic regurgitation was observed. Modified parasternal short axis view revealed aortic valve with two cusp (figure 1 and video 1). Parasternal long axis view showed an incomplete muscular VSD whose mouth was opened at diastole and closed in systole (figure 2 and video 2). Although high blood pressure is frequently encountered as primary hypertension, it is seen in young patients due to secondary causes such as CoA, pheochromocytoma and renal artery fibromuscular dysplasia. Coexistence of aortic coartation with BAV is a well known congenital anomaly. In this case severe stenosis in the proximal part of the descending aorta was detected in computed tomographic angiography (figure 3). Patient was referred to the cardiovascular surgeon for surgical intervention due to moderate aortic insufficiency and CoA.

Pacman heart (incomplete muscular ventricular septal defect) is extremely rare congenital deformity $(1,2)$, and combination of partial VSD, BAV and CoA occurring in the same patient has not been reported yet.

\section{References}

1. Mohan JC, Shukla M, Mohan V, et al. Parachute mitral valve and Pacman deformity of the ventricular septum in a middle-aged male.Indian Heart J. 2016 Sep;68 Suppl 2:S126-S130. doi: 10.1016/j.ihj.2015.09.012.

2. Pujol E, Morales M, Roelandt JR, et al., Partial ventricular septal defect (Pacman Heart).Eur J Echocardiogr. 2008 Mar;9(2):316-7. doi: 10.1093/ejechocard/jem068. 


\section{Figure and movie legends}

Figure 1A: Transthoracic two-dimensional echocardiography (modified parasternal short axis view) revealed aortic valve with two cusp. Ao = aortic valve, $\mathrm{LA}=$ left atrium, $\mathrm{RA}=$ right atrium, $\mathrm{RVOT}=$ right ventricular out-flow tract.

Figure 1B: In the modified parasternal long axis window, incomplete ventricular septal defect become more visible during diastole (yellaow arrow). Ao = aortic valve, $\mathrm{LA}=$ left atrium, $\mathrm{LV}=$ left ventricle.

Figure 1C: Computed tomography angiography shows severe stenosis at the beginning of the descending aorta (white arrow).

Movie S1: Video showing the aortic valve is bicuspid. Ao $=$ aortic valve, $\mathrm{LA}=$ left atrium, $\mathrm{RA}=$ right atrium, RVOT = right ventricular out-flow tract.

Movie S2: Modified parasternal long axis window showed incomplete ventricular septal defect, opened in diastole and closed during systole (asterix). Ao = aortic valve, $\mathrm{LA}=$ left atrium, $\mathrm{LV}=$ left ventricle.
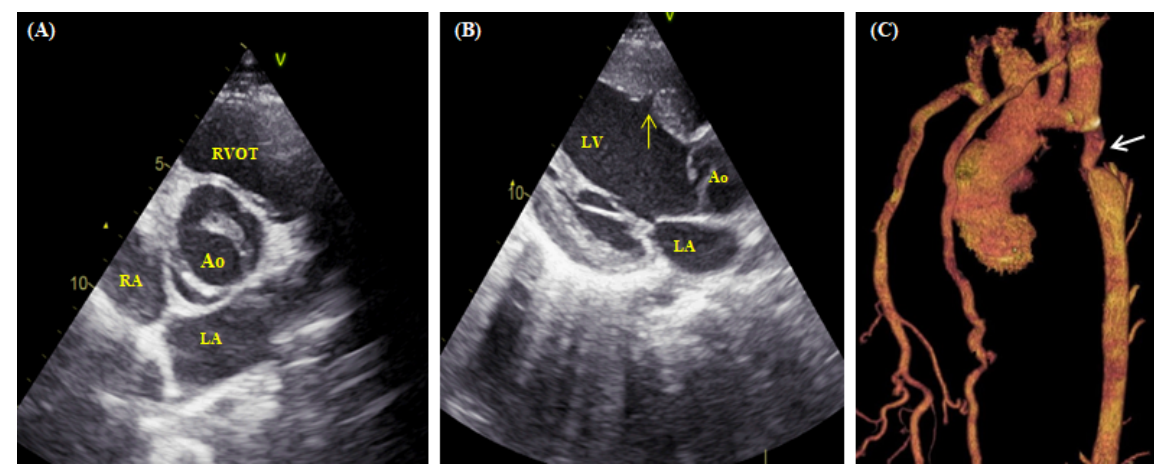